\title{
ABSENCE OF WORLD-SHEET AND SPACE-TIME ANOMALIES IN THE SEMI-COVARIANTLY QUANTIZED HETEROTIC STRING
}

\author{
Massimo Porrati ${ }^{1}$ and Peter van Nieuwenhuizen ${ }^{2}$ \\ Theory Division, CERN, Geneva, Switzerland
}

\begin{abstract}
The anomalies of the heterotic string in the semi light-cone gauge can be removed by adding suitable local order $\hbar$ terms to the local Weyl and spacetime (rigid) Lorentz transformation rules. The resulting quantum transformation rules exactly satisfy the spacetime Lorentz algebra. This removes the doubt, expressed in the literature, concerning the consistency of the heterotic string at the quantum level.
\end{abstract}

CERN-TH.6250/91

September 1991

\footnotetext{
${ }^{1}$ On leave of absence from Istituto Nazionale di Fisica Nucleare, Sezione di Pisa, Pisa, Italy.

${ }^{2}$ On leave of absence from Institute of Theoretical Physics, State University of New York at Stony Brook, Stony Brook, NY 11794, USA.
} 
The covariant quantization of superstrings (strings with manifest spacetime supersymmetry [1]) is still an unsolved problem. The positive results obtained for the superparticle cannot be carried over to the superstring in an obvious manner since the $\sigma$-dependence of the string leads to constraints which do not commute [2].

The light-cone quantization of the superstring yields $\hbar$-independent generators by the Noether procedure, which satisfy the rigid super-Poincaré algebra [3]. If the effective action were to be free from anomalies, one would expect that also in other gauges the quantum transformation rules, or at least their local part, would satisfy the same super-Poincaré algebra. Of course, these quantum transformation rules, which by definition leave the effective action invariant, may differ in general from the classical transformation rules by nonlocal loop corrections and further local corrections of order $\hbar$ and higher ${ }^{3}$.

As a step towards a fully covariant quantization of the superstring, there exists a partially covariant quantization scheme $[5,6]$, called semi light-cone quantization, in which the troublesome local fermionic $\kappa$ symmetry [7] is fixed at the classical level by the gauge choice $\Gamma^{+} \Theta=0$, while all other local symmetries are covariantly quantized. Here $\Gamma^{ \pm}=\left(\Gamma^{9} \pm \Gamma^{0}\right) / \sqrt{2}$ with $\left\{\Gamma^{\mu}, \Gamma^{\nu}\right\}=2 \eta^{\mu \nu}$ and $\eta^{\mu \nu}=\operatorname{diag}(-1,+1, . .,+1)$, while $\Theta$ is the 16-component Majorana-Weyl spacetime spinor. By integrating the world-sheet anomalies (corresponding to Einstein, Weyl and local Lorentz gauge invariance), the effective action was obtained. The world-sheet anomalies could be cancelled by adding a local counter term depending on the background values $u^{\mu}$ of the $d=10$ coordinate fields $X^{\mu}$. This effective action vanishes identically in the chiral sector with the 32 heterotic fermions, while in the other chiral sector with the $8 \Theta$ fields one finds $[8,9]$

$$
\Gamma^{e f f}=-\frac{d-26+8 / 2}{96 \pi} \hbar \int d^{2} x e R\left\{\frac{1}{e \nabla^{2}} e R^{+}+\log \nabla_{-} u^{+}\right\}
$$

with, of course, $d=10$. Here $R=R^{+}+R^{-}$, where $R^{+}=-2\left(d_{-} \omega_{+}+\omega_{+} \omega_{-}\right)$is a local Lorentz and Einstein scalar, and $d_{-}=e_{-}^{\alpha} \partial_{\alpha}$ while $\nabla_{-} u^{+}=d_{-} u^{+}$. The expression $e R\left(e \nabla^{2}\right)^{-1} e R^{+}$contains as nonlocal piece the chiral Polyakov action $2 \int d^{2} x \partial_{-}^{2} h_{++}\left[\partial_{-}\left(\partial_{+}\right.\right.$ $\left.\left.-h_{++} \partial_{-}\right)\right]^{-1} \partial_{-}^{2} h_{++}$and further local terms proportional to $h_{--}$. (The fields $h_{++}=$ $e_{+}^{-} / e_{-}^{-}$and $h_{--}=e_{-}^{+} / e_{+}^{+}$are local Lorentz and Weyl invariant combinations of the vielbein fields, and are related to the metric $g_{\alpha \beta}$ in the chiral gauge by $g_{+-}=\eta_{+-}=1$ and $g_{++}=2 h_{++}$). Since the expression between curly brackets in (1) is local Lorentz and Weyl invariant, the effective action is Einstein and local Lorentz invariant, but under Weyl transformations one has $\delta(e R)=e \nabla^{2} 2 \sigma$, and the ensuing Weyl anomaly only vanishes on the $u$-shell, where $\nabla_{+} \nabla_{-} u^{+}=\left(d_{+}-\omega_{+}\right) d_{-} u^{+}=0$. This fact will be important for what follows below.

The spacetime transformation rules acquire extra $\Theta$-dependent terms in order to maintain the gauge $\Gamma^{+} \Theta=0$. It turns out that the only contribution to spacetime anomalies comes from the local couterterm in (1), which shifts the anomalies from the world-sheet to the spacetime sector. Under rigid Lorentz transformations one has $\delta_{\Lambda} \nabla_{-} u^{+}=\Lambda_{-j} \nabla_{-} u^{j}+\Lambda_{-+} \nabla_{-} u^{+}$and

$$
\delta \Gamma^{e f f}=\frac{\hbar}{8 \pi} \int d^{2} x e R\left(\Lambda_{-+}+\Lambda_{-j} \frac{\nabla_{-} u^{j}}{\nabla_{-} u^{+}}\right) .
$$

\footnotetext{
${ }^{3}$ These local corrections may be viewed as counterterms proportional to the external source coupled to the transformation laws of the fields [4], as we will later show.
} 
In [9] an analysis of this result was made, starting from

$$
R=\nabla^{2}\left\{\log e_{-}^{-} e_{+}^{+}-\left(\partial_{+}-h_{++} \partial_{-}\right)^{-1} \partial_{-} h_{++}-\left(\partial_{-}-h_{--} \partial_{+}\right)^{-1} \partial_{+} h_{--}\right\} .
$$

It was argued that, if one were allowed to partially integrate (3), eq. (2) would vanish on the $u$-shell. On a generic world-sheet though, partial integration of (3) is non legitimate, in that the expression within curly brackets is only formally an Einstein and local Lorentz scalar, but not well-defined in general. As a consequence, partial integration gives rise to nonvanishing contributions, and these contributions would seem to make the heterotic string inconsistent at the quantum level.

We have reanalyzed this problem, and found that one can write $\delta \Gamma^{e f f}$ in a covariant manner proportional to the $u$ field equations. We shall show that one can add order $\hbar$ terms to the Weyl and rigid (spacetime) Lorentz transformations rules such that (i) all quantum anomalies are cancelled off-shell, (ii) the quantum Lorentz transformation rules contain compensating Weyl transformations and close on the rigid Lorentz algebra without any order $\hbar$ leftovers.

The expression in (2) can be written as follows

$$
\delta \Gamma^{e f f}=\frac{\hbar}{4 \pi} \int d^{2} x \frac{e}{\nabla_{-} u^{+}}\left(\nabla_{-} \nabla_{+}-\nabla_{+} \nabla_{-}\right) \Lambda_{-\mu} \nabla_{-} u^{\mu}
$$

since $R v_{-}=2\left(\nabla_{-} \nabla_{+}-\nabla_{+} \nabla_{-}\right) v_{-}$for a vector $v_{-}{ }^{4}$. By partially integrating the $\nabla_{+}$in $\nabla_{+} \nabla_{-}$, and the $\nabla_{-}$in $\nabla_{-} \nabla_{+}$, all terms become proportional to the $u$ field equations. Notice that here partial integration is legitimate, since in eq. (4) $\nabla_{-} u^{\mu}$ and $1 / \nabla_{-} u^{+}$ are bona fide local Lorentz vectors ${ }^{5}$. Boundary terms, present when the world-sheet has boundaries, are harmless. A further discussion of this point is presented in the concluding remarks. Normalizing the classical action for $X^{\mu}$ to $e X^{\mu} \nabla_{+} \nabla_{-} X_{\mu}$, we find that the anomalies in (4) can be removed by adding the following order $\hbar$ terms to the rigid Lorentz transformation rules of $X^{\mu}$

$$
\hat{\delta}_{\Lambda} X^{+}=0, \quad \hat{\delta}_{\Lambda} X^{-}=\frac{\hbar}{8 \pi} \Lambda_{-j} \frac{\nabla_{-}^{2} X^{j}}{\left(\nabla_{-} X^{+}\right)^{2}}, \quad \hat{\delta}_{\Lambda} X^{j}=-\frac{\hbar}{8 \pi} \Lambda_{-j} \frac{\nabla_{-}^{2} X^{+}}{\left(\nabla_{-} X^{+}\right)^{2}}
$$

Similarly the above mentioned off-shell Weyl anomaly can be removed by adding the following order $\hbar$ corrections to the Weyl transformation law for $X^{-}$

$$
\hat{\delta}_{\sigma} X^{-}=\frac{\hbar}{4 \pi} \frac{\nabla_{-} \sigma}{\nabla_{-} X^{+}} .
$$

We have now cancelled all world-sheet as well as spacetime anomalies, even off-shell and proceed to study their algebra at order $\hbar$.

We define new Lorentz transformation rules $\delta_{\Lambda}^{\prime}=\delta_{\Lambda}+\hat{\delta}_{\Lambda}+\hat{\delta}_{\sigma(\Lambda)}$, where the last term leaves open the possibility that we shall need a compensating Weyl transformation at order $\hbar$. We claim that for

$$
\sigma(\Lambda)=-\Lambda_{-j} \frac{\nabla_{-} X^{j}}{\nabla_{-} X^{+}}
$$

\footnotetext{
${ }^{4}$ Use $\nabla_{ \pm} v_{-}=d_{ \pm} v_{-}-\omega_{ \pm} v_{-}$and $\left(d_{+}-\omega_{+}\right) d_{-}=\left(d_{-}+\omega_{-}\right) d_{+}$.

${ }^{5}$ They are Einstein scalars, since we used flat world-sheet indices.
} 
these $\delta_{\Lambda}^{\prime}$ satisfy the spacetime Lorentz algebra at order $\hbar$. Although this can be verified straightforwardly, it may be helpful to give a few details. We want to show that on $X^{+}, X^{-}$and $X^{j}$ the following identity holds

$$
\left[\delta_{\Lambda}, \hat{\delta}_{\lambda}+\hat{\delta}_{\sigma(\lambda)}\right]+\left[\hat{\delta}_{\Lambda}+\hat{\delta}_{\sigma(\Lambda)}, \delta_{\lambda}\right]=\hat{\delta}_{[\lambda, \Lambda]}+\hat{\delta}_{\sigma([\lambda, \Lambda])}
$$

where $[\lambda, \Lambda]_{\mu}^{\nu}=\lambda_{\mu}^{\rho} \Lambda_{\rho}^{\nu}-\Lambda_{\mu}^{\rho} \lambda_{\rho}^{\nu}$. On $X^{+}$, for example, $\hat{\delta}_{\sigma}$ and $\hat{\delta}_{\Lambda}$ both vanish, hence the left hand side of (8) should vanish on $X^{+}$. One indeed finds $-\Lambda_{\mu}^{+} \hat{\delta}_{\lambda} X^{\mu}-\lambda \leftrightarrow \Lambda$. Since this is both antisymmetric in $\lambda, \Lambda$ and proportional to $\Lambda_{-j} \lambda_{-j}$ it indeed vanishes.

On $X^{j}$ the terms without $\hat{\delta}_{\sigma}$ in (8) leave a term

$$
-\frac{\hbar}{4 \pi} \lambda_{-j} \Lambda_{-k} \frac{1}{\nabla_{-} X^{+}} \nabla_{-}\left(\frac{\nabla_{-} X^{k}}{\nabla_{-} X^{+}}\right)-\lambda \leftrightarrow \Lambda,
$$

which is cancelled by the $\hat{\delta}_{\sigma}$ contributions, provided $\sigma$ is given by ( 7 ).

Finally, on $X^{-}$, one finds that omitting all $\hat{\delta}_{\sigma}$ in (8), on the left-hand side there is a contribution

$$
-\frac{\hbar}{4 \pi} \lambda_{-j} \Lambda_{-k} \frac{\nabla_{-}^{2} X^{j}}{\left(\nabla_{-} X^{+}\right)^{2}} \nabla_{-} X^{k}-\lambda \leftrightarrow \Lambda .
$$

The terms with $\hat{\delta}_{\sigma}$ in $(8)$ cancel this contribution, provided $\sigma(\Lambda)$ is again given by (7), and reconstruct the $\hat{\delta}_{\sigma([\lambda, \Lambda])}$ term on the left-hand side of $(8)$.

We conclude with some remarks

(i) Usually one tries to remove anomalies by adding a local counter term of order $\hbar$ to the classical action. The modified transformation rules correspond also to a local counter term as we now show.

The order $\hbar$ anomaly $\Delta$ can be obtained as

$$
\left(\frac{\delta \Gamma^{0}}{\delta \phi} \frac{\delta}{\delta K}+\frac{\delta \Gamma^{0}}{\delta K} \frac{\delta}{\delta \phi}\right) \Gamma^{1}=\Delta
$$

where the effective action is expanded as $\Gamma=\sum_{n} \hbar^{n} \Gamma^{n}$ and the $K$ are the sources for the symmetry variations of the fields $\phi$ [4]. The classical variation of $\phi$ is $\delta \Gamma^{0} / \delta K$. The first term in (11) is sometimes written as $\delta \Gamma^{0} / \delta \phi(\delta \phi \cdot \Gamma)^{1}$ and contains the one-loop corrections to $\delta \phi$. Usually one tries to add a $K$-independent local counterterm of order $\hbar$ to $\Gamma$, which cancels $\hbar \Delta$. However, one can also try to eliminate $\hbar \Delta$ by adding a local $K$-dependent counterterm. This counterterm corresponds to our order $\hbar$ modifications of the classical transformation laws, given in eq. (5) and (6), and reads $K \hat{\delta} \phi$.

Actually, we, and also the authors of refs. [8,9], only considered (11) at $K=0$. For general $K$, the $K$-dependent counterterms must obey the consistency conditions (8), which can be straightforwardly obtained from eq. (11). However, in our case, if one uses point splitting regularization, $(\delta \phi \cdot \Gamma)^{1}$ vanishes, as these one-loop corrections to $\delta \phi$ are proportional to $\operatorname{Tr}_{\mathrm{r}} \nabla_{+}^{-1}$. Hence, at one-loop, the quantum transformation rules are local. In other words, the only source of order $\hbar$ modifications to the transformation rules is the classical variation of the counterterm in (1).

(ii) If one would also admit fermionic background fields, one could repeat our analysis for rigid spacetime supersymmetry transformations.

(iii) In [10], Chu performed a Hamiltonian analysis of the supersymmetry generators 
of the Green-Schwarz superstring. She introduced extra fields, in order to have only first class constraints, but she found only closure up to a BRST transformation (her equation (23)).

(iv) Previously, the conformal (Weyl) anomaly of the Green-Schwarz superstring was shown to cancel in a path-integral approach by Carlip [6], and Kallosh and Morozov [5]. These latter authors did not diagonalize the $\Theta$-action by rescaling all the $\Theta$, rather, they first rewrote the $\Theta$ 's as two SU(4) spinors, and then integrated one of them out. The remaining $\Theta$ fields had an unusual kinetic action, and these authors claimed that the $\Theta$ fields contribute a coefficient $8 \times 2$, instead of $8 \times 1 / 2$ to the Weyl anomaly. We have rescaled all $\Theta$ 's equally, and taken these rescaled $\Theta$ 's as the basic variables in the path-integral. Our kinetic term is standard and yields a coefficient $8 \times 1 / 2$ in the $\Theta$ sector, see (1), but we need the local counter term in (1) to shift the anomalies to the spacetime sector. The authors in [6] and [5] did not consider spacetime anomalies.

(v) For the Green-Schwarz superstring $[1,3]$, the same cancellation of anomalies holds, but now in both chiral sectors.

(vi) Finally, we come back to the boundary terms mentioned below eq. (4). They are proportional to

$$
\int d^{2} x \partial_{\alpha}\left[\epsilon^{\alpha \beta} \Lambda_{-\mu} \frac{1}{\nabla_{-} u^{+}} \nabla_{-} \nabla_{\beta} u^{\mu}\right],
$$

where we used that $\nabla_{-}$and $\nabla_{\beta}$ cornmute when acting on the world-sheet scalar $u^{\mu}$. For closed strings one has boundary terms at $\tau= \pm \infty$, which vanish. For open strings, one has another term in eq. (12), with all $\nabla_{-}$replaced by $\nabla_{+}$, coming from the other chiral sector, and also boundaries at $\sigma=0,2 \pi$ for all $\tau$. In this case, the boundary terms vanish due to the open string boundary conditions $\partial_{\sigma} u^{\mu}=0$.

\section{References}

[1] M.B. Green and J.H. Schwarz, Phys. Lett. B136 (1984) 367;

D.J. Gross, J. Harvey, E. Martinec and R. Rohm, Phys. Rev. Lett. 54 (1985) 502; Nucl. Phys. B256 (1985) 253; B267 (1986) 75.

[2] See e.g. the review talk by W. Siegel at the conference "Strings and Symmetries," Stony Brook, NY (1991).

[3] M.B. Green and J.H. Schwarz, Nucl. Phys. B243 (1984) 285.

[4] C. Becchi, in "Les Houches 1983," (North-Holland 1984).

[5] R. Kallosh and A. Morozov, Int. Jou. of Mod. Phys. A3 (1988) 1943.

[6] S. Carlip, Nucl. Phys. B284 (1987) 365.

[7] W. Siegel, Phys. Lett. B128 (1983) 397.

[8] U. Kraemmer and A. Rebhan, Phys. Lett. B236 (1990) 255.

[9] F. Bastianelli, P. van Nieuwenhuizen and A. Van Proeyen, Phys. Lett. B253 (1991) 67.

[10] M. Chu, Nucl. Phys. B353 (1991) 538. 